\title{
Disloziertes Dexamethason-Implantat in der Vorderkammer: ein Fallbericht
}

\author{
Bujar Berisha (D) - Lukas Höflechner · Andreas Wedrich
}

Eingegangen: 27. August 2020 / Angenommen: 16. Dezember 2020 / Online publiziert: 18. Januar 2021

(c) Der/die Autor(en) 2021

\section{Zusammenfassung}

Hintergrund Ozurdex ${ }^{\circledR}$ (Allergan Pharmaceuticals Ireland, Westport, Co. Mayo, Irland) ist ein intravitreales Implantat von $700 \mathrm{mcg}$ Dexamethason, das für die Behandlung eines Makulaödems infolge eines retinalen Venenverschlusses, einer nichtinfektiösen posterioren Uveitis und einer diabetischen Makulopathie zugelassen ist. Erhöhter Augeninnendruck sowie Trübungen der kristallinen Linse sind bekannte Komplikationen. Seltener wird in der Literatur auch die unerwünschte Migration des Implantates in die Vorderkammer (VK) beschrieben. Laut Literatur erforderten die meisten Implantatdislokationen in die VK eine operative Entfernung oder Reposition in den Glaskörperraum. Wir berichten die erfolgreiche Repositionierung eines Ozurdex ${ }^{\circledR}$-Implantats durch die medikamentöse Erweiterung der Pupille.

Falldarstellung Eine 79-jährige Patientin entwickelte nach einer sekundären Intraokularlinsenimplantation ein persistierendes zystoides Makulaödem am linken Auge. Dieses wurde seit 3 Jahren mit intravitrealem Ozurdex ${ }^{\circledR}$ behandelt. Bei der geplanten Kontrolle 4 Wochen nach der letzten Behandlung wurde das Ozurdex ${ }^{\circledR}$-Stäbchen in der VK zwischen Iris und Optik der Vorderkammerlinse (VKL) festgestellt. Es erfolgte eine medikamentöse Erweiterung der Pupille, um das dislozierte Stäbchen zu mobilisieren. Die anschließende Spaltlampenuntersuchung und Fundoskopie zeigten eine Rücklagerung des Ozurdex ${ }^{\circledR}$-Implantates in dem Glaskörperraum.

Schlussfolgerungen Die Entfernung des Ozurdex ${ }^{\circledR}$ Stäbchens aus der VK ist notwendig, um eine Dekompensation des Hornhautendothels zu vermeiden.

B. Berisha $(\bowtie) \cdot$ L. Höflechner $\cdot$ A. Wedrich

Universitäts-Augenklinik, Medizinische Universität Graz,

Auenbruggerplatz 4, 8036 Graz, Österreich

bujar.berisha@medunigraz.at
Wir empfehlen vor einer chirurgischen Intervention einen Repositionsversuch des Implantats durch die medikamentöse Erweiterung der Pupille.

Schlüsselwörter Wanderung · Rücklagerung • Ozurdex · Glaskörper · Mydriasis

Anterior chamber migration of a dexamethasone implant: a case report

\section{Summary}

Background Ozurdex ${ }^{\circledR}$ (Allergan Pharmaceuticals Ireland, Westport, Co. Mayo, Ireland) is a $700 \mu \mathrm{g}$ dexamethasone intravitreal implant, approved for the treatment of macular edema due to retinal vein occlusion, non-infectious posterior uveitis and diabetic maculopathy. Increased intraocular pressure and lens opacification are known to be common complications. Less frequently, an anterior chamber dislocation of Ozurdex ${ }^{\circledR}$ has also been described. According to the literature, most transplant dislocations into the anterior chamber require surgical removal. This article reports the successful repositioning of an Ozurdex ${ }^{\circledR}$ implant by dilating the pupil with medicinal mydriasis.

Case presentation A 79-year-old woman developed persistent cystoid macular edema in the left eye after secondary intraocular lens implantation, which had been treated since March 2016 with intravitreal Ozurdex $^{\circledR}$. The patient underwent a planned examination 4 weeks after the last Ozurdex ${ }^{\circledR}$ implantation. Examination of the anterior chamber revealed an Ozurdex ${ }^{\circledR}$ rod located in the anterior chamber between the iris and the optic of the anterior chamber lens. A medicinal mydriasis of the pupil was performed to mobilize the dislocated Ozurdex ${ }^{\circledR}$. After 30 min the slit lamp examination showed that the dexamethasone implant was no longer visible in the 
anterior chamber. The subsequent funduscopy confirmed that the Ozurdex ${ }^{\circledR}$ implant was relocated in the vitreous cavity.

Conclusion The removal of the Ozurdex ${ }^{\circledR}$ implant from the anterior chamber is necessary to avoid corneal endothelial decompensation; however, prior to surgical intervention, it is recommended to attempt repositioning of the Ozurdex ${ }^{\circledR}$ implant by dilating the pupil by medicinal mydriasis.

Keywords Dislocation - Reposition - Ozurdex · Vitreous body $\cdot$ Mydriasis

\section{Hintergrund}

Ozurdex $^{\circledR}$ (Allergan Pharmaceuticals Ireland, Westport, Co. Mayo, Irland) ist ein Dexamethason-haltiges (700 mcg), biologisch abbaubares, stäbchenförmiges Implantat für die intravitreale Anwendung. Es ist $6 \mathrm{~mm}$ lang, hat einen Durchmesser von $0,46 \mathrm{~mm}$ und wird mittels eines speziellen 22-Gauge-Einmalapplikators in den Glaskörper injiziert. Die Wirkstofffreisetzung erfolgt durch Diffusion in 2 Phasen: eine anfängliche Phase hoher Wirkstoffabgabe und eine zweite, lange Phase mit geringer Wirkstoffabgabe, um eine langfristige Effektivität/Wirkstoffabgabe zu ermöglichen [1]. Zugelassen ist Ozurdex ${ }^{\circledR}$ für die Therapie des diabetischen Makulaödems, retinaler Venenastoder Zentralvenenverschlüsse sowie zur Anwendung bei nichtinfektiöser Uveitis bzw. Entzündung des posterioren Augensegments. Die Wirksamkeit und Sicherheit der intravitrealen Injektion von Ozurdex ${ }^{\circledR}$ konnte in diversen Studien bestätigt werden [2-4]. Die Anwendung von Dexamethason-Implantaten ist im Allgemeinen nebenwirkungsarm. Neben Komplikationen und Nebenwirkungen einer langfristigen und wiederholten Therapie wie einer Linsentrübung oder der Entwicklung eines sekundären Glaukoms [5] können auch Komplikationen durch die Implantation selbst, wie z.B. Hyposphagma, Endophthalmitis [6] oder unbeabsichtigte Injektion in die Linse auftreten [7]. Eine seltene Komplikation ist die Migration des Ozurdex ${ }^{\circledR}$-Implantats in die Vorderkammer (VK). Wir berichten über die Reposition des dislozierten Ozurdex $^{\circledR}$-Stäbchens in der VK durch die medikamentöse Erweiterung der Pupille.

\section{Falldarstellung}

Nach umfassender Aufklärung erfolgte die freie $\mathrm{Zu}$ stimmung der Patientin zur Teilnahme an diesem Fallbericht. Eine 79-jährige Frau wurde zur geplanten 4-Wochen-Kontrolle nach einer intravitrealen Injektion von Ozurdex ${ }^{\circledR}$ am linken Auge vorstellig. Im Jahr 2015 wurde bei der Patientin ein zystoides Makulaödem diagnostiziert, das nach einer Kataraktoperation am linken Auge bei kompliziertem postoperativem Verlauf mit Dislokation, Intraokularlinsen-Reposition und im weiteren Verlauf einer Linsenexplantation mit sekundärer Implantation einer Vorderkammerlinse (VKL) auftrat. Zu Beginn erfolgte eine topische Therapie mit Ketorolac-Augentropfen (Acular ${ }^{\circledR}$; Alleran Pharmaceuticals Ireland, Westport, Co. Mayo, Irland). Nach kurzer Zeit stellte sich jedoch eine Therapieresistenz auf die lokale Tropftherapie ein, worauf eine Therapie mit intravitrealen Injektionen von Glukokortikoiden (Triamcinolon) eingeleitet wurde. Im Jahr 2018 erfolgte erstmals eine intravitreale Implantation von Dexamethason $\left(\mathrm{Ozurdex}^{\circledR}\right)$. Bis auf einer leichte Augendruckerhöhung $(30 \mathrm{~mm} \mathrm{Hg})$, welche mit antiglaukomatösen Augentropfen (Bimatoprost $0,1 \mathrm{mg} / \mathrm{ml}$ ) zielführend therapiert werden konnte, traten bisher keine Nebenwirkungen und Komplikationen auf. Neben den genannten Augenerkrankungen bestanden bei der Patientin eine Keratoconjunctivitis sicca, allgemeininternistisch ein arterieller Hypertonus und eine koronare Herzkrankheit. Subjektiv hat sich der Seheindruck der Patientin seit der letzten Implantation nicht wesentlich geändert, 10 Tage vor der geplanten 4-Wochen-Kontrolle fiel der Patientin jedoch ein weißer Faden in der VK auf. Der Visus am linken Auge betrug ohne Korrektur 0,32, am rechten Auge 0,63. Die Untersuchung des vorderen Augenabschnittes des linken Auges ergab eine reizfreie Konjunktiva, an der Hornhaut zeigten sich diverse Striae und eine zarte Trübung. Die Pupille präsentierte sich durch die bei der Implantation der VKL durchgeführte Iridektomie gering nach temporal verzogen. Im Bereich der Pupille, mit Anteilen zwischen Iris und VKL, zeigte sich das dislozierte Ozurdex ${ }^{\circledR}$-Implantat (Abb. 1). Nach vorheriger Fotodokumentation erfolgte als erster Versuch eine Reposition durch medikamentöse Erweiterung der Pupille (Tropicamid 0,5\%/Phenylephrin 1\%) mit dem Ziel, das dislozierte Stäbchen zu mobilisieren. Bei einer erneuten Untersuchung an der Spaltlampe nach ca. 30 min und zwischenzeitlichem Aufwärtsblicken der Patientin konnte das Ozurdex ${ }^{\circledR}$ ohne eine chirurgische Intervention erfolgreich in den Glaskörperraum rückgelagert werden (Abb. 2). Die übrige ophthalmologische Untersuchung ergab einen Rück-

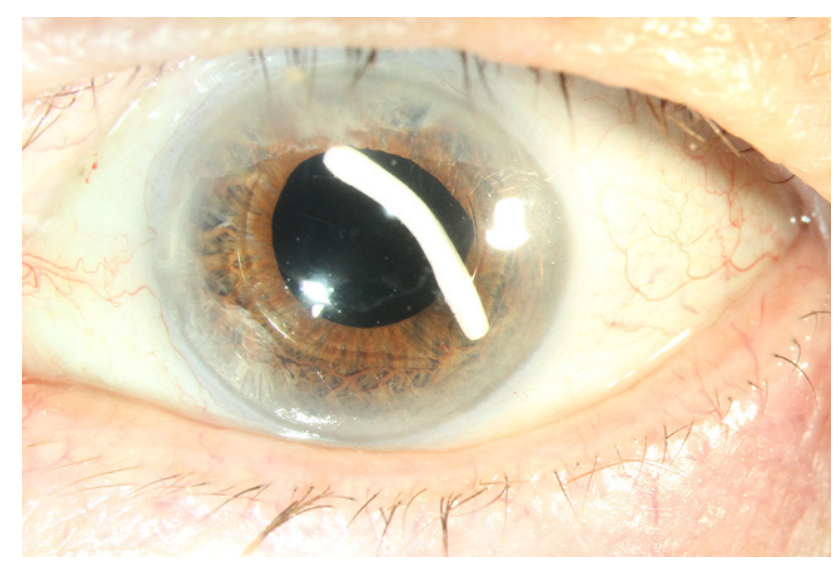

Abb. 1 Disloziertes Ozurdex ${ }^{\circledR}-S t a ̈ b c h e n$ in der Vorderkammer (VK) mit Anteilen zwischen Iris und Vorderkammerlinse (VKL) 


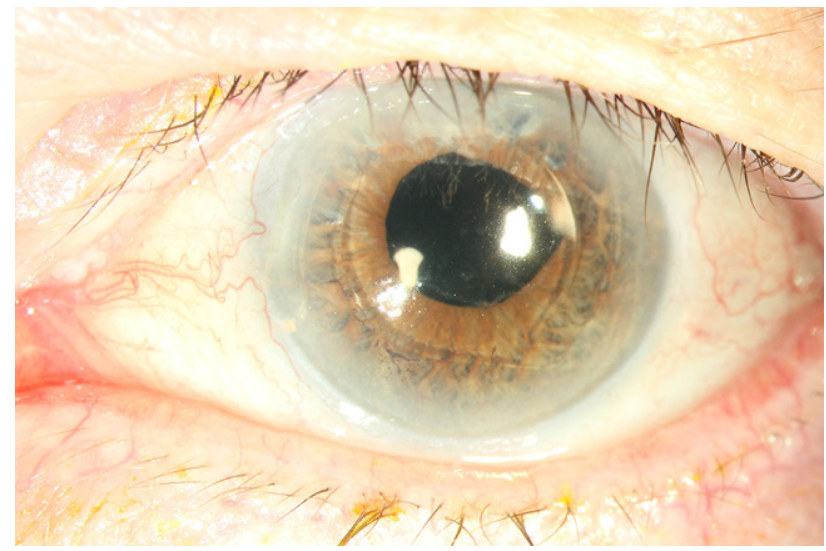

Abb. 2 Erfolgreiche Repositionierung des Ozurdex ${ }^{\circledR}$-Implantats nach medikamentöser Erweiterung der Pupille

gang der intraretinalen Zysten und eine Abnahme der Netzhautdicke.

Bei der Kontrolluntersuchung 3 Wochen später gab die Patientin weiterhin einen subjektiv gleichbleibenden Seheindruck an. Der erhobene Visus am linken Auge war unverändert bei 0,32. Anamnestisch kam es in der Zwischenzeit zu keinen weiteren Dislokationen des Ozurdex ${ }^{\circledR}$-Implantates in die VK. Die Untersuchung an der Spaltlampe ergab am linken Auge eine gestippte Hornhaut mit einer strichförmigen Trübung. Die VKL präsentierte sich in loco typico, die Pupille weiterhin nach temporal verzogen. Das Implantat von Ozurdex ${ }^{\circledR}$ ließ sich im Glaskörper inferior darstellen. Der Augendruck war unter der eingeleiteten Therapie gut reguliert. Ebenso wurde die konservierungsfreie benetzende Therapie erweitert.

Nach zwischenzeitlicher Umstellung auf Triesence (Wirktsoff Triamcinolon; Alcon, Fort Worth, TX, USA), welches sich ebenfalls in einer Kontrolluntersuchung in der VK darstellen ließ, erfolgte eine erneute Implantation von Ozurdex ${ }^{\circledR}$. Zehn Tage nach der Injektion wurde die Patientin wegen Schmerzen am linken Auge an der Augenklinik vorstellig. Die Untersuchung des vorderen Augenabschnitts ergab wiederum ein disloziertes Ozurdex ${ }^{\circledR}$-Implantat, welches zwischen der VKL und der Iris lag. Der Visus mit Korrektur betrug 0,16 am linken Auge, der Augendruck betrug $18 \mathrm{mmHg}$. Die Hornhaut zeigte eine zentrale oberflächliche Stippung. Ebenso wie bei der ersten Dislokation ließ sich das Stäbchen mit einer medikamentösen Mydriase und Aufwärtsblick für 30 min in den Glaskörperraum rücklagern. In der Kontrolluntersuchung 2 Wochen später gab die Patientin keine Beschwerden mehr an, die Schmerzen haben sich gebessert, und das Ozurdex ${ }^{\circledR}$-Implantat ließ sich wiederum im Glaskörper darstellen.

\section{Diskussion}

Die Luxation eines Ozurdex ${ }^{\circledR}$-Implantats aus dem Glaskörperraum in die VK ist eine seltene Kompli- kation, die häufiger bei Zustand nach Iridektomie, Kapselruptur, aphaken Augen oder vorderer Vitrektomie auftritt [6, 8-10]. In der größten Fallserie berichteten Khurana et al. [8] über 15 Patient*innen mit 18 Episoden von Dislokationen des DexamethasonImplantats in die VK. Die häufigsten beschriebenen Risikofaktoren waren die Vitrektomie und das Fehlen der Hinterkapsel. Des Weiteren können auch ungewöhnliche Körperhaltungen wie Bauchlage, körperliche Anstrengung sowie Langstreckenflüge zur Implantatdislokation in die VK beitragen [11, 12]. Das Ozurdex ${ }^{\circledR}$-Stäbchen in der VK kann zur Dekompensation des Hornhautendothels führen. Aus diesem Grund ist die Entfernung des Implantates oder Repositionierung in den Glaskörperraum notwendig [13].

Bei unserer Patientin haben wir primär ein möglichst konservatives Verfahren gewählt, um das Implantat in den Glaskörperraum zurückzulagern. Es erfolgte eine medikamentöse Erweiterung der Pupille mittels Tropicamid 0,5\%/Phenylephrin 1\%-Augentropfen, um das dislozierte Stäbchen zu mobilisieren. Als Nächstes wurde die Patientin angewiesen, die nächsten 30 min nach medikamentöser Mydriase den Kopf etwas nach hinten zu strecken und die Augen im Aufwärtsblick zu halten. Die erneute Untersuchung an der Spaltlampe zeigte, dass das Ozurdex ${ }^{\circledR}$-Implantat erfolgreich in den Glaskörperraum rückgelagert werden konnte.

Die Rücklagerung des Implantats im Glaskörperraum durch die medikamentöse Erweiterung der $\mathrm{Pu}$ pille haben zum ersten Mal Kishore et al. [14] probiert. Wichtig zu erwähnen ist, dass sich das OzurdexStäbchen sowohl in unserem Fall als auch bei Kishore et al. zwischen der VKL und der Iris befand. Nach unserem Wissen hat die Pupillenerweiterung zur Reposition des Implantates bisher nur in diesen 2 Fällen funktioniert.

Andere Forscher haben sich für engmaschige Observanz entschieden und das Stäbchen in der VK belassen [15, 16]. Bei Zafar et al. [15] ragte das Ozurdex ${ }^{\circledR}$ zwischen der Iris und der Optik in die VK herein, aber berührte die Hornhaut nicht. In diesem Fall wurde das Ozurdex ${ }^{\circledR}$ ohne Komplikationen aufgelöst. Bei Kocak et al. [16] migrierte das Ozurdex ${ }^{\circledR}$-Implantat in die hintere Kapsel und löste sich dort ohne Komplikationen auf. Wie bei Kishore et al. [14] und Zafar et al. [15] hat das Implantat auch bei unserer Patientin keinen direkten Kontakt mit der Hornhaut gehabt. Das könnte der Grund sein, dass es in keinem dieser Fälle zu einem akuten Hornhautödem kam.

In der Literatur sind bisher vorwiegend chirurgische Techniken zur Reposition [11, 12, 17] oder Entfernung des Implantats [18] beschrieben. Pacella et al. [12] berichten die erfolgreiche Repositionierung eines Ozurdex ${ }^{\circledR}$-Implantats in den Glaskörperraum durch mechanische Mobilisierung des Stäbchens mit einer 30-Gauge-Nadel und anschließende Injektion einer isotonen Kochsalzlösung in die VK. Nguyen und Wolfesberger [19] haben eine 23-Gauge-Nadel und ei- 
ne Spritze verwendet, um das dislozierte Stäbchen aus der VK zu aspirieren. In beiden Fällen erfolgte der Eingriff unter lokaler Tropfanästhesie in der Spaltlampe. Zum Schutz des Hornhautendothels wurde ein Viskoelastikum verwendet. Des Weiteren wurde auch ein Non-Touch-Technik zur Entfernung des Ozurdex ${ }^{\circledR}$ Stäbchens beschrieben. Durch eine Hornhautinzision wurde die VK mit Viskoelastikum aufgefüllt. Als Nächstes wurde die Kanüle weiter über das Ozurdex ${ }^{\circledR}$ Implantat vorgeschoben und eine „Welle“ mit Viskoelastikum erzeugt, um das Stäbchen in Richtung der Hornhautinzision zu bewegen [20].

Die Entscheidung über die Methode für die Entfernung oder Rücklagerung des Dexamethason-Implantats sollte in Abhängigkeit von der Position und dem Verhältnis des Implantats zu den anderen intraokularen Strukturen sowie der Anatomie des Auges bei bereits durchgeführten operativen Eingriffen getroffen werden.

Die konservative Reposition des Implantats mittels medikamentöser Erweiterung der Pupille hat den Vorteil, dass sie einfach durchzuführen ist sowie eine zeit- und ressourcenschonende Methode darstellt. Bei Erfolg können ein invasiver Eingriff und die damit verbundenen Risiken und Komplikationen vermieden werden. Ist diese Methode nicht erfolgreich, besteht immer noch die Möglichkeit eines chirurgischen Eingriffes.

\section{Schlussfolgerungen}

Die Ozurdex ${ }^{\circledR}$-Therapie zeigt sich in der klinischen Routine als nebenwirkungsarmes Therapieverfahren. Eine Dislokation des Implantates aus dem Glaskörperraum in die VK ist eine seltene, aber mögliche Komplikation. Insbesondere ist bei Patient*innen mit einer VKL, bei Zustand nach Iridektomie, Kapselruptur oder vorderer Vitrektomie Vorsicht geboten. Die Entfernung des Ozurdex ${ }^{\circledR}$-Stäbchens aus der VK ist notwendig, um eine Dekompensation des Hornhautendothels $\mathrm{zu}$ vermeiden. Wir empfehlen allerdings, vor einer chirurgischen Intervention, einen Repositionsversuch von Ozurdex ${ }^{\circledR}$ durch die medikamentöse Erweiterung der Pupille.

Funding Open access funding provided by Medical University of Graz.

\section{Einhaltung ethischer Richtlinien}

Interessenkonflikt B. Berisha, L. Höflechner und A. Wedrich geben an, dass kein Interessenkonflikt besteht.

Ethische Standards Alle beschriebenen Untersuchungen am Menschen oder an menschlichem Gewebe wurden gemäß der Deklaration von Helsinki von 1975 (in der aktuellen, überarbeiteten Fassung) durchgeführt. Von allen beteiligten Patienten liegt eine Einverständniserklärung vor. Ein Votum einer Ethikkommission war nicht nötig (klinischer Fallbericht).
Open Access Dieser Artikel wird unter der Creative Commons Namensnennung 4.0 International Lizenz veröffentlicht, welche die Nutzung, Vervielfältigung, Bearbeitung, Verbreitung und Wiedergabe in jeglichem Medium und Format erlaubt, sofern Sie den/die ursprünglichen Autor(en) und die Quelle ordnungsgemäß nennen, einen Link zur Creative Commons Lizenz beifügen und angeben, ob Änderungen vorgenommen wurden.

Die in diesem Artikel enthaltenen Bilder und sonstiges Drittmaterial unterliegen ebenfalls der genannten Creative Commons Lizenz, sofern sich aus der Abbildungslegende nichts anderes ergibt. Sofern das betreffende Material nicht unter der genannten Creative Commons Lizenz steht und die betreffende Handlung nicht nach gesetzlichen Vorschriften erlaubt ist, ist für die oben aufgeführten Weiterverwendungen des Materials die Einwilligung des jeweiligen Rechteinhabers einzuholen.

Weitere Details zur Lizenz entnehmen Sie bitte der Lizenzinformation auf http://creativecommons.org/licenses/by/4. $0 /$ deed.de.

\section{Literatur}

1. Chang-Lin JE, Attar M, Acheampong AA, Robinson MR, Whitcup SM, Kuppermann BD, et al. Pharmacokinetics and pharmacodynamics of a sustained-release dexamethasone intravitreal implant. Invest Ophthalmol Vis Sci. 2011;52:80-6.

2. HallerJA, BandelloF, BelfortRJr, BlumenkranzMS, GilliesM, Heier J, et al. Dexametasone intravitreal implant in patients with maculer edema related to branch or central retinal vein occlusion twelve month study results. Ophthalmology. 2011;118(12):2453-60.

3. Bellocq D, Korobelnik JF, Burillon C, et al. Effectiveness and safety of dexamethasone implants for post-surgical macular oedema including Irvine-Gass syndrome: the EPISODIC study. Br J Ophthalmol. 2015;99(7):979-83.

4. He Y, Ren XJ, Hu BJ, Lam WC, Li XR. A meta-analysis of the effect of a dexamethasone intravitreal implant versus intravitrealanti-vascularendothelialgrowth factor treatmentfor diabetic macular edema. BMC Ophthalmol.2018;18:121.

5. Meyer LM, Schönfeld CL. Secondary glaucoma afterintravitreal dexamethasone $0.7 \mathrm{mg}$ implant in patients with retinal vein occlusion: a one-year follow-up. J Ocul Pharmacol Ther. 2013;29(6):560-5.

6. Schmitz K, Maier M, Clemens CR, Höhn F, Wachtlin J, Lehmann F, et al. German retinal vein occlusion group. Reliability and safety of intravitreal ozurdex injections. The ZERO study. Ophthalmologe. 2014;111:44-52.

7. Coca-Robinot J, Casco-Silva B, Armadá-Maresca F, GarcíaMartínez J. Accidental injections of dexamethasone intravitreal implant (ozurdex) into the crystalline lens. Eur J Ophthalmol.2014;24(4):633-6.

8. Khurana RN, AppaSN, McCannelCA, et al. Dexamethasone implant anterior chamber migration: risk factors, complications, and management strategies. Ophthalmology. 2014;121(1):67-71.

9. Kang H, Lee MW, Byeon SH, et al. The clinical outcomes of surgical management of anterior chamber migration of a dexamethasone implant (ozurdex ${ }^{\circledR}$ ). Graefes Arch Clin Exp Ophthalmol.2017;255:1819-25.

10. Röck D, Bartz-Schmidt KU, Röck T. Risk factors for and management of anterior chamber intravitreal dexamethasone implant migration. BMCOphthalmol.2019;19(1):120. 
11. Malclès A, Janin-Manificat H, Yhuel Y, et al. Anterior chamber migration of intravitreal dexamethasone implant (ozurdex ${ }^{\circledR}$ ) in pseudophakic eyes: report of three cases. J Fr Ophtalmol.2013;36(4):362-7.

12. Pacella F, Agostinelli E, Carlesimo SC, Nebbioso M, Secondi R, Forastiere M, et al. Management of anterior chamber dislocation of a dexamethasone intravitreal implant: a case report. J Med Case Rep. 2016;10:282.

13. Madi HA, Morgan SJ, Ghosh S. Corneal graft failure due to migration of ozurdex ${ }^{\mathrm{TM}}$ implant into the anterior chamber. AmJ Ophthalmol Case Rep. 2017;8:25-7.

14. Kishore SA, Schaal S. Management of anterior chamber dislocation of dexamethasone implant. Ocul Immunol Inflamm. 2013;21(1):90-1.

15. Zafar A, Aslanides IM, Selimis V, Tsoulnaras KI, Tabibian D, Kymionis GD. Uneventful anterior migration of Intravitreal ozurdex implant in a patient with Iris-sutured Intraocular lens and descemet stripping automated endothelial keratoplasty. Case Rep Ophthalmol.2018;9:149-54.

16. Kocak N, Ozturk T, Karahan E, Kaynak S. Anterior migration of dexamethasone implant in a pseudophakic patient with intact posterior capsule. Indian J Ophthalmol. 2014;62(11):1086-8. https://doi.org/10.4103/0301-4738. 146763.

17. Vela JI, Crespi J, Andreu D. Repositioning of dexamethasone intravitreal implant (ozurdex ${ }^{\circledR}$ ) migrated into the anterior chamber. Int Ophthalmol. 2012;32:583-4.

18. Stelton CR, Townsend J, Peterson LT, et al. Surgical management of anterior chamber migration of a dexamethasone intravitreal implant. Ophthalmic Surg Lasers Imaging Retina. 2015;46(7):756-9.

19. Nguyen TL, Wolfensberger TJ. Management of anterior chamber dislocation of a dexamethasone implant. Klin Monbl Augenheilkd.2019;236(4):412-4.

20. Rahimy E, Pitcher JD III, Abbey AM, Garretson BR, Haller JA. No-touch removal of anterior segment-migrated dexamethasoneimplant. Retina.2015;35(11):2414-6.

Hinweis des Verlags Der Verlag bleibt in Hinblick auf geografische Zuordnungen und Gebietsbezeichnungen in veröffentlichten Karten und Institutsadressen neutral. 